\title{
DISEÑO E INCLUSIÓN DE HERRAMIENTASY ACTIVIDADES EN EL AULA VIRTUAL BAJO LA PLATAFORMA MOODLE, EN PROCESOS DE ENSEÑANZA - APRENDIZAJE DE LA QUÍMICA ${ }^{1}$
}

\author{
Rafael Alberto Solórzano* / dqu.rsolorzano@pedagogica.edu.co \\ Javier R. Arias* / holatodoscomo@hotmail.com \\ Jenny Paola Marín* / tormetork@yahoo.es \\ Juan C. Suárez ${ }^{\star}$ / dqu_jsuarez669@pedagogica.edu.co \\ Juan C. Vargas* / black_hawk_ct@hotmail.com \\ Fabián C. Corredor / dqu.fcorredor@pedagogica.edu.co
}

\section{Resumen}

Las Tecnologías de la Información y la Comunicación conocidas como TIC, juegan un papel importante en los procesos de Aprendizaje, en particular el Aula Virtual. El presente artículo muestra los logros obtenidos en el diseño e inclusión de herramientas y actividades en el Aula Virtual bajo la plataforma Moodle, como parte del proyecto de Práctica Pedagógica y Didáctica en la I.E.D. Rafael Bernal Jiménez. A partir de una observación-acción participativa se diseña una serie de actividades, recursos y evaluaciones soportadas en la plataforma virtual: www.casquetedeesferaperforada.com, con el fin de incorporar a profesores y estudiantes del ciclo 3, 4 y 5 del área de Ciencias, en el uso adecuado de las tecnologías, apoyados en referentes teóricos que favorecen su incorporación en Ambientes de Aprendizaje con un manejo apropiado de las TIC (Tecnologías de la Información y la Comunicación) y su migración hacia las TAC (Tecnologías para el Aprendizaje y el Conocimiento).

\section{Palabras Claves}

Ambientes de aprendizaje, Aula virtual, Evaluación, casquetedeesferaperforada, TIC, TAC, TEP². 


\section{Introducción}

Las comúnmente denominadas TIC - Tecnologías de la Información y la Comunicación - juegan un papel importante en los procesos de Aprendizaje, en particular el Aula Virtual, la cual, cumple una función de apoyo en la labor del profesor como mediador para la construcción de conocimiento. Allí, "es indispensable tener claro qué tipo de educación deseamos impulsar y cómo se puede favorecer el enfoque educativo", (Galvis, P. 2001), por tal motivo, la práctica pedagógica I y II llevada a cabo en la Institución Educativa Distrital Rafael Bernal Jiménez se fundamenta en el uso de las TIC y su migración hacia las TAC con el fin de fortalecer Ambientes Favorables menos condicionantes en el proceso de Aprendizaje.

La educación debe trascender las fronteras físicas de la escuela e ir más allá del contexto cotidiano escolar. Así como las TIC han logrado insertarse en los campos de la comunicación, lograr incluir dichas tecnologías en procesos de aprendizaje y conocimiento es el nuevo reto de su uso, tanto en la escuela como fuera de ella.

El presente trabajo, enfatiza su diseño en la evaluación, factor utilizado para incentivar el uso del Aula virtual.

La evaluación juega un papel importante en el proceso de formación, convirtiéndose en un regulador que orienta al estudiante al reconocimiento de sus errores y permite el ajuste en las ideas para ser acomodadas o reparadas (Chi, P. 2003). La evaluación sin supervisión (evaluación fuera del colegio), es un primer paso para entender la participación del estudiante en su proceso responsable y autónomo de formación, por ello se adecuan las pruebas de Estado tipo SABER, en el aula virtual, como un recurso para que cada estudiante identifique su nivel de conceptualización en las temáticas propias del Plan de Estudios.

\section{Problema}

La profesión docente busca el mejoramiento del Sistema Educativo. Uno de sus campos es el diseño de herramientas didácticas eficientes, para ser vinculadas en ambientes virtuales de aprendizaje, en ésta dirección, los estudiantes del ciclo 3, 4 y 5 del área de Ciencias naturales, a través del uso del Aula Virtual identificarán y aumentarán su nivel de comprensión de aquellas temáticas propias del Plan de Estudios de cada uno de los programas asignados a la Práctica, lo que lleva a la pregunta: ¿Qué tipo de actividades y herramientas pueden ser vinculadas al Aula Virtual para favorecer el desarrollo y evaluación de contenidos específicos del área de Ciencias naturales en el I.E.D Rafael Bernal Jiménez?

\section{Objetivo General}

Implementar ambientes de aprendizaje, apoyados en las TIC a través del Aula Virtual "www.casquetedeesferaperforada.", encaminados al desarrollo, comprensión y evaluación formativa de los estudiantes de la I.E.D Rafael Bernal Jiménez, Jornada Mañana.

\section{Objetivos Específicos}

- Seleccionar actividades y recursos disponibles en el Aula virtual, que permitan la conformación de Ambientes de Aprendizajes favorables y agradables para desempeños eficientes de los estudiantes.

- Diseñar herramientas evaluativas con contenidos específicos bajo la estructura del plan de estudios de la institución.

\section{Marco Teórico}

Una de las inquietudes asociadas a éste proyecto es la relacionada con el uso pertinente y coherente de las TIC en procesos de Aprendizaje.

El veloz y gran desarrollo en los sistemas computacionales han generado nuevos conceptos que se incorporaron al uso de las tecnologías de la información y la comunicación - TIC. El acceso y uso de dichas tecnologías son estudiadas con detenimiento en el sector educativo de donde emergen términos como TAC (Tecnologías para el Aprendi- 
zaje y la Comunicación) y TEP (Tecnologías para el Empoderamiento y la Participación) cuyo objeto central es el aprendizaje y crecimiento social (Sociedad aumentada) (Reig, 2012). No se trata de desarrollar habilidades para manejar herramientas tecnológicas de moda; por esto es fundamental trabajar para cambiar la forma de pensar el uso de dichas herramientas.

La implementación del Aula Virtual como uno de los productos del avance de las TIC, se constituye en un recurso importante en procesos que van más allá de la habilidad en su uso y manejo, convirtiéndose en medios para el ascenso de la heteronomía (individuo gobernado por otros), hacia la autonomía responsable (individuo capaz de tomar decisiones).

\section{Moodle}

Moodle es una plataforma multiusuario que permite el montaje de recursos y actividades, allí es posible trabajar a partir de tres (3) categorías diferentes: como administrador, como profesor y como estudiante. Ofrece igualmente, una gran variedad de acciones como blogs, chats, foros, wikis, etc. De otro lado, la plataforma también facilita la creación de diferentes contenidos en diversos formatos como enlaces, materiales interactivos cuestionarios, lecciones, glosarios y tareas entre otros. (Arratia, 2009).

\section{TIC, TAC y TEP}

Considerando la formación inicial de profesores de ciencias naturales y su importante inmersión en el mundo de las TIC, es claro que las condiciones sociales que predominan en las comunidades del siglo XXI requieren de una formación continua y participativa del profesor en ésta dirección, lo que hace indispensable conocer y manejar las nuevas herramientas tecnológicas y científicas con las que se disponen, sin embargo, no se puede dejar de lado las concepciones que direccionan estas innovaciones tecnológicas, para hacer de las clases y el trabajo autónomo de los estudiantes una dinámica más activa y constructiva; por lo tanto "el profesor debe actuar como agente generador de situaciones y condiciones para que el alumnado aprenda y no se limite a almacenar datos estériles e inconexos" (Domingo y Fuentes, 2010).

La alfabetización tecnológica no puede dejar de lado el aprendizaje y el conocimiento, por lo que las TAC (tecnologías para el aprendizaje y el conocimiento), en la actualidad se convierten en una herramienta de fácil acceso para estudiantes y profesores.

El concepto TAC creado por Vivancos, permite explicar las nuevas posibilidades que las tecnologías brindan a la educación, cuando éstas dejan de usarse como un elemento instrumental. Su nueva función pasa a que "el contexto sociotecnológico genere un nuevo modelo de escuela que responda a las necesidades formativas de los ciudadanos" (Castañeda y Adell, 2013).

En cuanto a las TEP, Dolors Reig (2012), plantea que para la primera mitad del siglo XXI, la tendencia "en educación se trata de educar en la sociedad aumentada (socioeconomía). Propone la evolución a partir de una "educación pasiva" hacia lo que ella denomina "aprendizaje aumentado" lo que implica centrar la atención en el aprendizaje autónomo y aprovechar la potencia de la web para formar nuevos individuos conectados en forma permanente a la inteligencia colectiva responsable y transformadora. Seremos más grandes si estamos juntos". (Reig, 2012).

\section{Evaluación y usos pedagógicos}

La evaluación se ha convertido en el principal instrumento que tiene el profesor para verificar el desempeño en los procesos educativos: La evaluación es una de las herramientas educativas más poderosas para promover el aprendizaje efectivo, pero debe usarse de manera adecuada. No hay evidencia de que aumentar la cantidad de pruebas lo reforzará; en lugar de ello, el foco debe estar en ayudar a que los profesores usen la evaluación como parte de la enseñanza y del aprendizaje, de tal modo que aumente los logros de los estudiantes. (Altablero, 2008). 
La educación mediada por computador se utiliza para compartir información con los estudiantes. En la multimedia basada en el computador, se integran impresos, audio, video y procesador en un sistema de información y datos de fácil acceso, ejemplo son los Materiales Educativos Computacionales (MEC). (Galvis, P. 2001).

Varias son las ventajas de los computadores: facilitan la regulación del proceso de aprendizaje, la interacción de los individuos y grupos, y el acceso a recursos humanos y técnicos difícil de obtener por otros medios. (Galvis, P. 2001).

Una clasificación predominante es la que propone Robert T., cuando dice que los computadores pueden servir: como tutor, como herramienta y como aprendiz, esto quiere decir, como medio de enseñanza-aprendizaje (educación apoyada con computador), como herramienta de trabajo (educación complementada con computador) y como objeto de estudio (educación acerca de la computación). (Galvis, P. 2001).

\section{Diseño Metodológico}

Se enmarca dentro de una investigación de corte cualitativo con componentes cuantitativos debido al uso del ingreso al Aula Virtual. Como parte del diseño se hace necesario describir las fases de la estrategia a seguir, como referencia para posteriores trabajos que permitan consolidar el uso adecuado de las TIC y su migración a las TAC.

En el Anexo 1 se encuentra la tabla que detalla las etapas y la descripción del proceso que se lleva a cabo en la IED Rafael Bernal Jiménez Jornada Mañana.

\section{Resultados- Análisis}

Los análisis y resultados respectivos se evidencian a partir de dos momentos, el primero, corresponde al primer bimestre de 2012, en el que se realiza una prueba piloto con estudiantes del ciclo 5 y se desarrolla una actividad en el aula virtual. El segundo momento, corresponde al primer bimestre del 2013, donde se vinculan estudiantes de los ciclos 3 y 4, para que inicien el manejo no sólo de actividades, sino de evaluaciones en el marco de la virtualidad, con los siguientes datos:

\section{Primer momento}

En el primer semestre de 2013 se aplica una encuesta con siete (7) preguntas sencillas, teniendo en cuenta que otros cursos ya se habían incorporado al proyecto y el objeto era ampliar la población para aplicar y desarrollar actitudes frente al uso adecuado de las TIC.

\begin{tabular}{|c|c|c|c|c|}
\hline Resultado / curso & 801 & 901 & 1102 & total \\
\hline Registrados & 54 & 23 & 19 & 96 \\
\hline No registrados & 4 & 16 & 4 & 24 \\
\hline
\end{tabular}

Tabla 1. Registro estudiantes segundo bimestre del 2012

Estudiantes
\begin{tabular}{|l|c|} 
Realizaron la actividad & Total \\
\hline No realizaron la actividad & 6 \\
\hline Total & 19 \\
\hline
\end{tabular}

Tabla 2. Visualización de videos - curso 1102 (Ciclo 5)

\begin{tabular}{|c|c|}
\hline & Estudiantes \\
\hline Total registrados & 36 \\
\hline Total participantes & 19 \\
\hline Problemas de acceso & 3 \\
\hline Problemas con el sistema & 1 \\
\hline
\end{tabular}

Tabla 3. Acceso y uso de los estudiantes curso 801 (Ciclo 4)

\section{Segundo Momento}

Se busca obtener información acerca de la accesibilidad a internet y sus múltiples utilidades con los estudiantes del colegio Rafael Bernal Jiménez correspondiente a los ciclos II, IV y V; los resultados son los siguientes: 


\begin{tabular}{|c|c|c|} 
Grados & Ciclo & $\begin{array}{c}\text { Estudiantes registra- } \\
\text { dos }\end{array}$ \\
\hline Sexto & 3 & 119 \\
\hline Séptimo & 3 & 44 \\
\hline Noveno & 4 & 120 \\
\hline Decimo & 5 & 76 \\
\hline Once & 5 & 51 \\
\hline \multicolumn{2}{|c|}{ Total } & 410 \\
\hline
\end{tabular}

Tabla $\mathrm{N}^{\circ}$ 4. Estudiantes registrados en el ciclo 3,4 y 5

\begin{tabular}{|c|c|c|}
\hline Sí & No & Total \\
\hline 241 & 136 & 377 \\
\hline $64 \%$ & $36 \%$ & $100 \%$ \\
\hline
\end{tabular}

Tabla $\mathrm{N}^{\circ}$ 5. Dispone de computador con Internet en la casa

\begin{tabular}{|c|c|c|c|} 
Facilidad & Dificultad & $\begin{array}{c}\text { No la } \\
\text { maneja }\end{array}$ & Total \\
\hline 297 & 60 & 20 & 377 \\
\hline $79 \%$ & $16 \%$ & $5 \%$ & $100 \%$ \\
\hline
\end{tabular}

Tabla $\mathrm{N}^{\circ}$ 6. Maneja el Internet con facilidad o con dificultad, o no lo maneja

\begin{tabular}{|c|c|c|}
\hline Si & No & Total \\
\hline 99 & 278 & 377 \\
\hline $26 \%$ & $74 \%$ & $100 \%$ \\
\hline
\end{tabular}

Tabla $N^{\circ} 7$. Alquila tiempo de Internet en cabinas.

\begin{tabular}{|c|c|c|c|c|}
\hline $\begin{array}{c}5-15 \\
\min \end{array}$ & $\begin{array}{c}15-30 \\
\min \end{array}$ & $\begin{array}{c}30-60 \\
\min \end{array}$ & $\begin{array}{c}60 \text { min } \\
0 \text { mas }\end{array}$ & Total \\
\hline 15 & 63 & 95 & 204 & 377 \\
\hline $4 \%$ & $17 \%$ & $25 \%$ & $54 \%$ & $100 \%$ \\
\hline
\end{tabular}

Tabla N`8. Tiempo dedicado a Internet por parte de los estudiantes

\begin{tabular}{|c|c|c|c|}
\hline Facebook & YouTube & Google & Otros \\
\hline 209 & 103 & 56 & 214 \\
\hline $36 \%$ & $18 \%$ & $9 \%$ & $37 \%$ \\
\hline
\end{tabular}

Tabla N9. Qué tipo de Páginas o Redes sociales regularmente maneja

\begin{tabular}{|c|c|c|c|}
\hline$\$ 1.000$ & $\$ 2.500$ & $\$ 10.000$ & Total \\
\hline 203 & 150 & 24 & 377 \\
\hline $74 \%$ & $19 \%$ & $7 \%$ & $100 \%$ \\
\hline
\end{tabular}

Tabla $N^{\circ} 10$. Cuánto dinero gasta diariamente en el uso de Internet

\begin{tabular}{|c|c|c|}
\hline Si & No & Total \\
\hline 320 & 57 & 377 \\
\hline $85 \%$ & $15 \%$ & $100 \%$ \\
\hline
\end{tabular}

Tabla $N^{\circ}$ 11. Usaría Internet para mejorar su desempeño académico

\section{Evaluaciones por ciclo}

A continuación se describe una muestra de las diversas pruebas realizadas en cada ciclo, representado por los cursos respectivos.

Para el grado 1102, se asignan las claves para el registro en el aula virtual, (tabla No 1). Como primera actividad del curso se propone la revisión de cuatro videos que introduce a los estudiantes en la comprensión de la geometría molecular para compuestos orgánicos, (tabla No 2).

Para este grado, se trabaja sobre una serie de preguntas que ponen a prueba lo revisado en clase, las preguntas empleadas son muy similares al desarrollo en clase, en esta prueba se busca evidenciar problemas en el acceso a la plataforma y a su vez mostrar problemas con temáticas ya tratada. De esta prueba se obtienen datos, en cuanto al acceso a la página y a los resultados arrojados: De los estudiantes que presenta, la prueba tan sólo 
cuatro (4) aprueban, el resto la reprueba, se maneja un puntaje de 0 a 100. El curso obtuvo un promedio de 53.38 puntos del total evaluado.

\section{Habilidades lectoras}

Con el fin de identificar las interpretaciones que hacen los estudiantes a las lecturas suministradas por medio del aula virtual, es conveniente identificar las dificultades que tienen en la interpretación de textos para de ellos obtener respuestas. Se diseña una prueba en la cual la mayor parte de las actividades son fragmentos cortos de textos o lecturas, de los cuales, y con base en lo visto en clase pueden dar respuestas acertadas. En este curso se desarrolla, una prueba estructurada según las pruebas SABER, evaluando la temática especifica de enlace químico.

\section{Aplicación de la Encuesta}

Con miras a justificar el proceso de los estudiantes al usar el aula virtual para realizar actividades como talleres, encuestas y otros procesos pertinentes a la evaluación, se realiza un test de actitudes frente al uso de las TIC, que permite, según el estudio, desarrollar y complementar los espacios de aprendizaje existentes y llevarlos a un nivel más estructurado.

En el semestre 2013-I, se aplica una encuesta de siete preguntas sencillas en donde se busca obtener información acerca de la accesibilidad a internet y sus múltiples utilidades para los estudiantes del I.E.D Rafael Bernal Jiménez correspondiente a los ciclos IV y V, con los siguientes resultados:

En el grado 901 se evidencia que el 59\% realizan el registro e ingresan a la prueba; el $41 \%$ restante no ingresan por varias causas, entre las que se encuentran: no poseen un computador, no les llega el correo de confirmación para acceder a la plataforma, no quieren realizar la prueba; las anteriores causas fueron mencionadas por los alumnos que no ingresan, esto en parte muestra un reporte no muy alentador, lo que permite inducir que hace falta trabajar más en el diseño de herramientas virtuales que despierten el interés del alumnado.
En el grado 1102 se puede observar que más del $50 \%$ de los estudiantes realizan un primer acercamiento en el desarrollo de actividades en el aula virtual, allí se obtienen resultados alentadores, sin embargo, en la confrontación de estos resultados con las actividades desarrolladas posteriormente en el aula de clases, se ve que la actividad no cumplió con los objetivos propuestos, para lo cual se deben hacer mejoramientos en las actividades y evaluaciones que se propongan en dicho espacio con el fin de optimizar los resultados y obtener una mejor disposición del estudiante hacia estas herramientas virtuales.

Con respecto al curso 801, se observa que la moda en cuanto a los resultados obtenidos en el aula virtual esta entre los 50 y 60 puntos de los 100 evaluados. Estas repuestas reflejan poca atención por parte de los estudiantes para acceder a estas herramientas, ya que sólo se cuenta con el 52\% del curso como participantes, por otro lado los resultados no son alentadores, en la medida que se esperan mejores respuestas, conociendo que las mismas preguntas ya han sido desarrolladas. Los estudiantes no poseen buena interpretación de textos, tienen problemas para analizar situaciones en textos, de 36 estudiantes tan sólo el 19 \% del curso logra aprobar el examen, correspondiendo a 7 estudiantes. En otras pruebas realizadas posteriormente se evidencian problemas de redacción y mala ortografía.

En la aplicación de la encuesta se encuentra:

- El $64 \%$ de los estudiantes del I.E.D Rafael Bernal Jiménez manifiestan tener computador con internet en sus casas, mientras que el 36\% no tienen acceso a internet desde sus casas presentando esa dificultad al momento de navegar por el mismo.

- El $79 \%$ de los estudiantes del I.E.D Rafael Bernal Jiménez manejan con facilidad el internet, mientras el $16 \%$ se le dificulta y un $5 \%$ no la maneja.

- El 26\% de los estudiantes del I.E.D Rafael Bernal Jiménez alquila internet en cabinas, mientras que el $74 \%$ no alquila. 
- El 85\% de los estudiantes del I.E.D Rafael Bernal Jiménez manifiestan que usarían el internet para mejorar su desempeño académico, mientras que el $15 \%$ no lo usarían.

Así mismo, se encuentra que un 36\% de los estudiantes manejan el internet para visitar Facebook.

\section{Conclusiones}

- El aula virtual es una herramienta que le sirve al docente para el seguimiento y acompañamiento más detallado del estudiante, le brinda igualmente información para sugerir refuerzo en temáticas cuyos resultados no sean los esperados.

- Las TIC son herramientas de apoyo para ampliar la virtualización en la conformación de ambientes de aprendizaje y posibilita procesos centrados en aprendizajes.
- Es preciso trabajar sobre las habilidades lectoras que amplíen la capacidad de interpretación de todos los estudiantes, para poder implementar adecuadamente las actividades desde el aula virtual.

- A pesar de la innovación que trae consigo la utilización de las tecnologías de la información, aún en la actualidad el trabajo presencial sigue arrojando mejores resultados que el pensado por las TIC.

- Se encuentra que un alto porcentaje de los estudiantes tiene acceso a internet, lo que facilita la integración de los mismos al manejo del aula virtual.

- Es necesario orientar a los estudiantes al buen manejo de herramientas tecnológicas, ya que se encuentra que un alto porcentaje de los estudiantes manejan el internet para otro tipo de actividades.

\section{Bibliografía}

Altablero. (2008). Usar la evaluación en el aula para mejorar. Ministerio de Educación Nacional. Altablero. Recuperado el 13 de Agosto de 2012, de: http://www.mineducacion.gov.co/1621/article-162385.html.

Arratia, O., y otros. (2009). Innovación en Docencia Universitaria con Moodle: casos prácticos. España. Gamma.

Chi, P. (2003) The process and challenges of conceptual change. En: Limón, M. and Mason, L. (2003). Reconsidering conceptual change:

Domingo, M. y Fuentes, M. (2010). Innovación educativa: experimentar con las TIC y reflexionar sobre su uso. Revista de Medios y educación, Número 36, Enero. Pág. 171-180.
Galvis, P. (2001), ingeniería de software educativo, capítulo 1, Educativa e informática educativa, Universidad de los Andes, Bogotá- Colombia.

Pensa, D. y Sabulsky, G. (1999) "Sobre el uso de los media en los procesos de enseñanza" en Tercer Simposio Internacional de Educación a Distancia: La educación a distancia y el aprendizaje abierto: Aportes para la construcción de un nuevo paradigma educativo.

Reig, D. (2012). Socionomía. Grupo Planeta, Barcelona, España. 


\section{ANEXO 1}

\section{Etapas Diseño Metodológico}

\begin{tabular}{|c|c|}
\hline Etapa & Descripción \\
\hline Test sobre uso de las TIC & $\begin{array}{l}\text { Esta prueba permite informar sobre la actividad extracurricular de } \\
\text { los estudiantes con respecto al tiempo que dedican a internet y si } \\
\text { de alguna forma se les dificulta ingresar a esta plataforma, esto } \\
\text { se aplica a todos los grados donde se implementa la plataforma } \\
\text { virtual. Como herramienta de consulta y evaluación, se programan } \\
\text { las diferentes actividades, en las salas de informática del colegio y } \\
\text { en espacios extracurriculares, como la casa, café internet, etc. }\end{array}$ \\
\hline Estudio de Referentes & $\begin{array}{l}\text { Se lleva a cabo una detallada revisión bibliográfica en donde se } \\
\text { abordan temas teóricos sobre aula virtual, evaluación, uso de TIC y } \\
\text { ambientes de aprendizaje. }\end{array}$ \\
\hline $\begin{array}{l}\text { Orientación sobre la } \\
\text { importancia del aula virtual }\end{array}$ & $\begin{array}{l}\text { Contextualización con el cotidiano de la institución educativa, en } \\
\text { busca de un nuevo desarrollo pedagógico desde la virtualidad. } \\
\text { Capacitación en el manejo de software educativo. }\end{array}$ \\
\hline $\begin{array}{l}\text { Indicaciones para estudiantes } \\
\text { del ciclo } 3,4 \text { y } 5\end{array}$ & $\begin{array}{l}\text { Charlas informativas a cargo de los profesores en formación, } \\
\text { con el fin de introducir al estudiante en el manejo de las TIC, } \\
\text { particularmente de la plataforma Moodle. }\end{array}$ \\
\hline $\begin{array}{c}\text { Contextualización de las } \\
\text { herramientas del aula virtual }\end{array}$ & $\begin{array}{l}\text { Subir a la plataforma diversas actividades empleando todas las } \\
\text { herramientas que puede ofrecer Moodle, para familiarizar al } \\
\text { estudiante con el sistema virtual. }\end{array}$ \\
\hline Inducción & $\begin{array}{l}\text { Enmarcados en el tercer período académico estudiantil, aplicar } \\
\text { una prueba piloto de } 21 \text { preguntas para analizar la aceptación y } \\
\text { dificultades al momento de emplear este medio. }\end{array}$ \\
\hline Resultados Prueba aplicada & $\begin{array}{l}\text { Al realizar la prueba en la plataforma Moodle, se obtienen } \\
\text { resultados puntuales conforme a las particularidades de la prueba, } \\
\text { los cuales fueron llevados a una posterior comparación. }\end{array}$ \\
\hline
\end{tabular}

